\title{
The potential of intra-articular injection of chondrogenic-induced bone marrow stem cells to retard the progression of osteoarthritis in a sheep model
}

\begin{abstract}
In recent years, the use of bone marrow mesenchymal stem cell (BMSC) implantation has provided an alternative treatment for osteoarthritis. The objective of this study is to determine whether or not an intra-articular injection of a single dose of autologous chondrogenic induced BMSC could retard the progressive destruction of cartilage in a surgically induced osteoarthritis in sheep. Sheep BMSCs were isolated and divided into two groups. One group was cultured in chondrogenic media containing (Ham's F12:DMEM, 1:1) FD + 1\% FBS + 5 ng/ml TGF63 + $50 \mathrm{ng} / \mathrm{ml} \mathrm{IGF-1} \mathrm{(CM),} \mathrm{and} \mathrm{the} \mathrm{other} \mathrm{group} \mathrm{was} \mathrm{cultured} \mathrm{in} \mathrm{the} \mathrm{basal} \mathrm{media,}$ FD $+10 \%$ FBS $(\mathrm{BM})$. The procedure for surgically induced osteoarthritis was performed on the donor sheep 6 weeks prior to intra-articular injection into the knee joint of a single dose of BMSC from either group, suspended in $5 \mathrm{ml} \mathrm{FD}$ at density of 2 million cells $/ \mathrm{ml}$. The control groups were injected with basal media, without cells. Six weeks after injection, gross evidence of retardation of cartilage destruction was seen in the osteoarthritic knee joints treated with CM as well as BM. No significant ICRS (International Cartilage Repair Society) scoring was detected between the two groups with cells. However macroscopically, meniscus repair was observed in the knee joint treated with CM. Severe osteoarthritis and meniscal injury was observed in the control group. Interestingly, histologically the $\mathrm{CM}$ group demonstrated good cartilage histoarchitecture, thickness and quality, comparable to normal knee joint cartilage. As a conclusion, intra-articular injection of a single dose of BMSC either chondrogenically induced or not, could retard the progression of osteoarthritis (OA) in a sheep model, but the induced cells indicated better results especially in meniscus regeneration.
\end{abstract}

Keyword: Chondrogenic induction; Stem cell therapy; Intra-articular injection; Osteoarthritis; Aging; Tissue engineering 